\title{
siRNA-mediated AMPKa1 subunit gene PRKAA1 silencing enhances methylmercury toxicity in HEK293 cells
}

\author{
Gi-Wook Hwang', Mayumi Tobita', Tsutomu Takahashi' ${ }^{1}$, Shusuke Kuge ${ }^{1,2}$, Kayoko Kita' ${ }^{1,3}$ \\ and Akira Naganuma ${ }^{1}$ \\ ${ }^{1}$ Laboratory of Molecular and Biochemical Toxicology, Graduate School of Pharmaceutical Sciences, Tohoku \\ University, Aoba-ku, Sendai, Miyagi 980-8578, Japan \\ ${ }^{2}$ Department of Microbiology, Tohoku Pharmaceutical University, Komatsushima, Aoba-ku, Sendai, \\ Miyagi 981-8558, Japan \\ ${ }^{3}$ Laboratory of Toxicology, Faculty of Pharmaceutical Sciences, Teikyo University, 1091-1 Sagamiko-cho, \\ Sagamihara, Kanagawa 229-0195, Japan
}

(Received February 27, 2010; Accepted April 3, 2010)

\begin{abstract}
The environmental pollutant methylmercury is a potent neurotoxin. The mechanisms of toxicity and biological defense remain largely unknown. We found that inhibiting the expression of PRKAA1 (AMPK $\alpha 1$ ), an activated subunit of AMP-activated protein kinase (AMPK), increased susceptibility of HEK293 cells to methylmercury toxicity. Treatment of the cells with AICAR (5-aminoimidazole4-carboxamide-1- $\beta$-D-ribofuranoside), an AMPK activator, reduced the methylmercury toxicity. Here, we suggest for the first time that the activation (phosphorylation) of AMPK may play an important role in reducing the toxicity of methylmercury.
\end{abstract}

Key words: Methylmercury, AMP-activated protein kinase, PRKAA1 (AMPK $\alpha 1$ ), AICAR

\section{INTRODUCTION}

Methylmercury causes serious disorders of the central nervous system (Clarkson and Magos, 2006). Antioxidative enzymes and antioxidants, for example, reduce the toxicity of methylmercury (Naganuma et al., 1998; Usuki et al., 2001; Watanabe et al., 2009). According to several reports, the ubiquitin proteasome system, a selective proteolysis pathway, plays an important role in reducing the toxicity of methylmercury (Furuchi et al., 2002; Hwang et al., 2002, 2005, 2007 and 2009). However, other defense mechanisms might exist against the toxicity of methylmercury (Hwang et al., 2007; Lee et al., 2009). Hence, we introduced an siRNA library into HEK293 cells to investigate factors that make cells highly susceptible to methylmercury toxicity via decreased gene expression.

\section{MATERIALS AND METHODS}

\section{Transduction of the siRNA library into HEK293 cells and microarray analysis}

HEK293 cells, a human embryonic kidney cell line, were maintained in Dulbecco's modified eagle's medium (DMEM) with 10\% heat-inactivated fetal bovine serum (FBS), $100 \mathrm{IU} / \mathrm{ml}$ penicillin, and $100 \mathrm{mg} / \mathrm{ml} \mathrm{strep-}$ tomycin in a humidified $5 \% \mathrm{CO}_{2}$ atmosphere at $37^{\circ} \mathrm{C}$. A GeneNet ${ }^{\mathrm{TM}}$ human $50 \mathrm{~K}$ siRNA library (System Biosciences, Mountain View, CA, USA) that contained about 50,000 human genes was prepackaged in pseudoviral particles using the FIV lentiviral expression system. The detailed protocol for the transduction of the siRNA library is available from System Biosciences (Hwang et al., 2007). Briefly, HEK293 cells $\left(2 \times 10^{6}\right.$ cells/plate $)$ were plated on five $10-\mathrm{cm}$ plates and cultured in $10-\mathrm{ml}$ aliquots of medium. After incubation for $24 \mathrm{hr}$, the culture medium was removed and the cells were infected with the pseudoviral particles $\left(4 \times 10^{6}\right.$ ifu/plate $)$ in plates containing $5 \mathrm{ml}$ of DMEM/3\% FBS with polybrene (final: $6 \mu \mathrm{g} / \mathrm{ml}$ ). After incubation for $12 \mathrm{hr}$, the culture medium was removed from each plate and replaced by $10 \mathrm{ml}$ of $\mathrm{DMEM} / 3 \%$ FBS without polybrene and then cultured for an additional $60 \mathrm{hr}$. The siRNA library-transduced cells (5 $\times 10^{6}$ cells) were plated into seven 10 -cm plates containing $10 \mathrm{ml}$ of DMEM/10\% FBS medium. After incubation

Correspondence: Akira Naganuma (E-mail: naganuma@mail.pharm.tohoku.ac.jp) 
for $24 \mathrm{hr}, 3 \mu \mathrm{M}$ methylmercury chloride was added and the cells were cultured for an additional $48 \mathrm{hr}$. Total RNA was isolated with TRIzol ${ }^{\circledR}$ (Invitrogen, Carlsbad, CA, USA) according to the manufacturer's protocol. Then, the purified RNA was reverse transcribed by M-MLV reverse transcriptase (Invitrogen) using a cDNA synthesis primer ( 5 ' - T G C A T G T C G C T A T G T G T T C T G G G A $\left.-3^{\prime}\right)$ specific to a sequence in the pFIV-H1 siRNA expression vector (System Bioscience). For microarray hybridization, the amplified siRNA targets were labeled with biotin and used as hybridization targets with GeneChip ${ }^{\circledR}$ arrays (HG_U133 set; Affymetrix, Santa Clara, CA, USA) employing standard protocols.

\section{Construction of an siRNA expression plasmid}

For cloning into the pFIV-H1 siRNA expression vector, two DNA oligonucleotide pairs were employed: (PRKAA 1-1: 5' - GATCCGTCACGATAACTTATGAGAGAAGTAAAGCTTCCTGTCAGACTTTGCTTCTCTTATAAGTTATTGTGATTTTTG -3' AND 5'- AATTCAAAAATCACAATAACTTATAAGAGAAGCAAAGTCTGACAGGAAGCTTTACTTCTCTCATAAGTTATCGTGACG -3'; AND PRKAA1-2: 5' - GATCCGCCTGTGATAAGCGCT$\underline{\text { TACTCCAGAATGCTTCCTGTCAGA CATTTT- }}$ GGAGTAAGTGCTTGTCACAGGTTTTTG -3' and 5' - AATTCAAAAACCTGTGACAAGCACTTACTCCAAAATGTCTGACAGGAAGCATTCTGGAGTAAGCGCTTATCACAGGCG -3'), which encoded siRNAs that targeted the 4,095-4,121 and 1,354-1,380 regions of the gene PRKAA1, respectively. The oligonucleotides were annealed, phosphorylated by T4 DNA polynucleotide kinase (Takara, Shiga, Japan), and then ligated into the pFIV-H1 siRNA expression vector. The sequences of oligonucleotide inserts were verified by using an automated sequencer. Plasmid DNA was transfected with LipofectamineTM 2000 reagent (Invitrogen) according to the manufacturer's protocols.

\section{Confirmation of knockdown by real-time PCR or immunoblotting}

For confirmation of the knockdown with the siRNA expression vectors expressing PRKAA1-1 and PRKAA12 siRNA, cells were lysed in TRIzol ${ }^{\circledR}$ reagent in order to isolate total RNA. First strand cDNA synthesis was performed using the PrimeScrip ${ }^{\mathrm{TM}}$ RT reagent kit (Takara). We performed real-time PCR reactions (Bio-Rad, iCycler $^{\mathrm{TM}}$ thermal cycler) with the following primers: PRKAA1-F, 5' - CCAACTATGCTGCACCAGAA -3' and PRKAA1-R, 5' - TGGCACATGGTCATCATCA -3' for the PRKAA1 gene; and GAPDH-F, 5'-CGGGAAGCTT-
GTCATCAATGG-3' and GAPDH-R, 5'-GGCAGTGATGGCATGGACTG-3' for the GAPDH gene. The fold decreases in PRKAA1 mRNA levels were determined from standard curves after calibration of the assay. For immunoblotting, cells were fixed in $20 \%$ trichloroacetic acid on ice for $30 \mathrm{~min}$ and then washed five times with cold PBS. The cell pellet was solubilized with lysis buffer (1\% SDS, 45 mM HEPES-Na pH 7.5). Cell lysates were separated by SDS-polyacrylamide gel electrophoresis (SDS-PAGE), transferred to an Immobilon-P membrane (Millipore, Bedford, MA, USA), and visualized using PRKAA1 (Upstate, Lake Placid, NY, USA)- or PRKAA2 (Cell Signaling, Danvers, MA, USA)-specific antibodies.

\section{RESULTS AND DISCUSSION}

An siRNA expression plasmid library against approximately 50,000 human genes was introduced into HEK293 cells using lentivirus. These siRNA-expressing cells were cultured for $48 \mathrm{hr}$ in the presence of $3 \mu \mathrm{M}$ methylmercury chloride, a sufficient concentration to inhibit the growth of normal cells by about $10 \%$. Subsequently, total RNA was extracted for microarray analysis using, as a probe, the PCR product specifically amplified from the sense strand of the siRNA sequence. The signal intensity of the probe obtained in this analysis indicates the percentage of living cells into which each siRNA was introduced. The results of the microarray analysis demonstrated that the signal intensity of the probe targeting the PRKAA1 gene was markedly reduced by treatment with methylmercury (data not shown). Then, two kinds of siRNA targeting different base sequences were synthesized and separately introduced into HEK293 cells, resulting in a susceptibility to methylmercury toxicity higher than that for the control cells (Fig. 1A). Both siRNAs markedly reduced the mRNA (data not shown) and protein levels of PRKAA1 compared with those of the control cells (Fig. 1B). These results suggest that inhibition of expression of PRKAA1 caused increased susceptibility of cells to methylmercury toxicity.

PRKAA1 is a catalytic subunit of AMPK (AMP-activated protein kinase) involved mainly in the maintenance of the homeostasis of intracellular energy metabolism, etc. (Hardie, 2005; Kahn et al., 2005). AMPK is a heterotrimeric protein kinase consisting of $\alpha$ subunits $(\alpha 1$ and $\alpha 2)$ exhibiting kinase activity and $\beta$ subunits $(\beta 1$ and $\beta 2)$ and $\gamma$ subunits $(\gamma 1, \gamma 2$, and $\gamma 3)$ involved in the control of the $\alpha$ subunits. PRKAA1 corresponds to the $\alpha 1$ subunit of AMPK (Carling, 2004). AMPK is activated (phosphorylated) by an increased ratio of intracellular AMP/ATP and various stresses (Marsin et al., 2002; Hardie, 2004; 

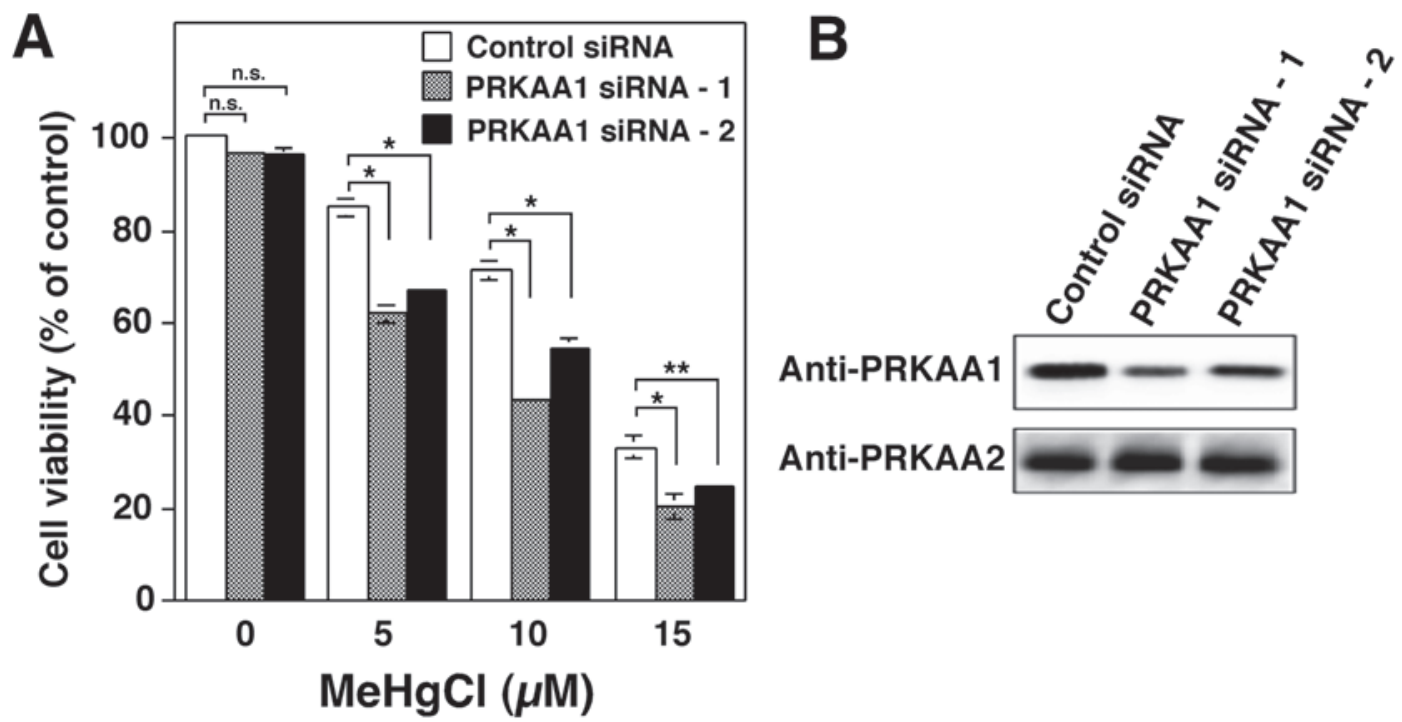

Fig. 1. Effects of knockdown of PRKAA1 on HEK293 cell sensitivity to methylmercury toxicity. (A) HEK293 cells $\left(2 \times 10^{4}\right.$ cells), that had been stably transfected with PRKAA1 siRNA, were plated on 96-well plates and cultured in $100 \mu 1$ aliquots of medium. After incubation for $24 \mathrm{hr}$, methylmercury chloride $(\mathrm{MeHg})$ was added and cells were cultured for a further 24 hr. Cell viability was determined by treating cells with Alamer Blue (Biosource, Camarillo, CA, USA). Each point and bar represents the mean value and S.D. of the results from three cultures, respectively. Data represents the mean \pm S.D. of three replicates. ${ }^{*} P<0.001$ as compared to control cells; ${ }^{* *} P<0.005$ as compared to control cells; n.s., not significant. Data were analyzed using the Student's $t$-test. (B) Levels of PRKAA1 protein in HEK293 cells transfected with PRKAA1 siRNA were analyzed by immunoblotting. Levels of PRKAA2 protein, the other component of AMPK, served as a loading control.

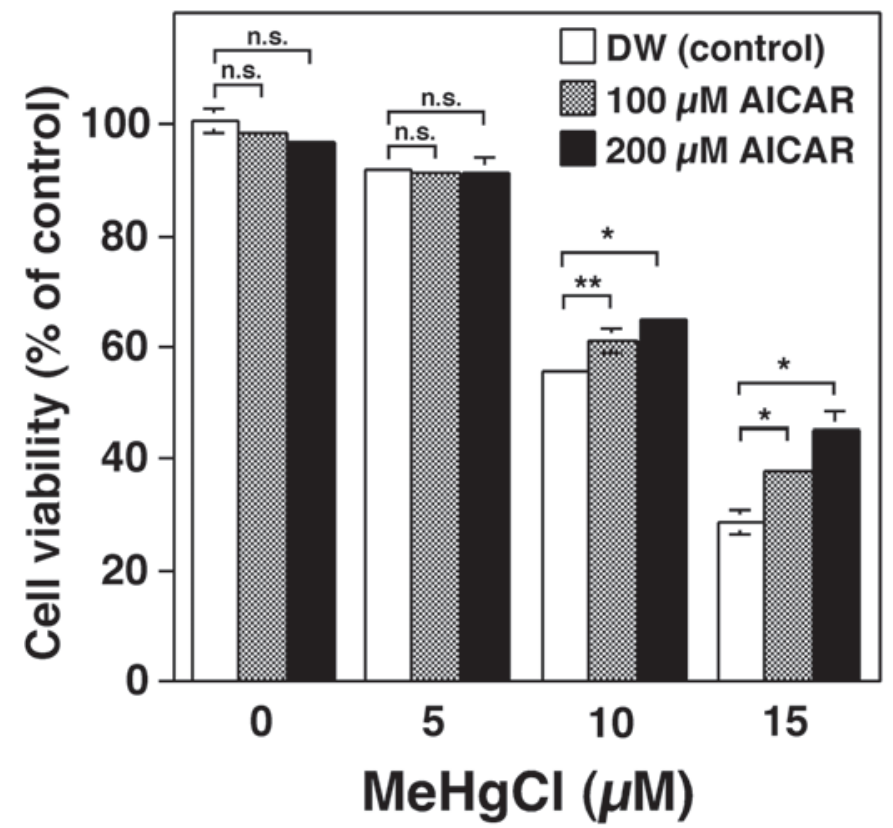

Fig. 2. Effects of AMPK activator (AICAR) on HEK293 cell sensitivity to methylmercury toxicity. HEK 293 cells $\left(2 \times 10^{4}\right.$ cells $)$ were pretreated or not with AICAR $(100 \mu \mathrm{M}$ or $200 \mu \mathrm{M})$, which had been dissolved in DW (distilled water), and $3 \mathrm{hr}$ later exposed to methylmercury chloride $(\mathrm{MeHg})$ at the indicated concentrations. Data represents the mean \pm S.D. of three replicates. $* P<0.005$ as compared to control cells; $* * P<0.01$ as compared to control cells; n.s., not significant. For further details, see the legend to Fig. 1A. 
Sanders et al., 2007). In cells carrying PRKAA1 siRNA, AMPK activity is presumed to be suppressed. Hence, decreased AMPK activity may make cells highly susceptible to methylmercury toxicity. The effects of treatment with AICAR (5-aminoimidazole-4-carboxamide- 1 - $\beta$-Dribofuranoside), an activator of AMPK, on the susceptibility to methylmercury toxicity were examined. The toxicity of methylmercury (at the higher levels tested: 10 and $15 \mu \mathrm{M} \mathrm{MeHgCl}$ ) was significantly reduced depending on AICAR concentration (Fig. 2). Thus, the signaling, including AMPK activation, may function in vivo as a novel defense mechanism against the toxicity of methylmercury. However, there has been no report that AMPK functions as an energy sensor to reduce environmental pollutants including methylmercury. In the future, detailed studies on the relationship between AMPK phosphorylation and methylmercury should elucidate the mechanism of toxicity of methylmercury and the biological defense mechanism.

\section{ACKNOWLEDGMENT}

This work was supported by a Grant-in-Aid for Scientific Research (S) from the Japan Society for the Promotion of Science.

\section{REFERENCES}

Carling, D. (2004): The AMP-activated protein kinase cascade--a unifying system for energy control. Trends Biochem. Sci., 29, $18-24$.

Clarkson, T.W. and Magos, L. (2006): The toxicology of mercury and its chemical compounds. Crit. Rev. Toxicol., 36, 609-662.

Furuchi, T., Hwang, G.W. and Naganuma, A. (2002): Overexpression of the ubiquitin-conjugating enzyme Cdc34 confers resistance to methylmercury in Saccharomyces cerevisiae. Mol. Pharmacol., 61, 738-741.

Hardie, D.G. (2004): The AMP-activated protein kinase pathway-new players upstream and downstream. J. Cell. Sci., 117, 54795487.

Hardie, D.G. (2005): New roles for the LKB1-->AMPK pathway. Curr. Opin. Cell Biol., 17, 167-173.

Hwang, G.W., Furuchi, T. and Naganuma, A. (2002): A ubiquitinproteasome system is responsible for the protection of yeast and human cells against methylmercury. FASEB J., 16, 709-711.

Hwang, G.W., Sasaki, D. and Naganuma, A. (2005): Overexpression of Rad23 confers resistance to methylmercury in Saccharomyces cerevisiae via inhibition of the degradation of ubiquitinated proteins. Mol. Pharmacol., 68, 1074-1078.

Hwang, G.W. (2007): Ubiquitin-proteasome system as a factor that determine the sensitivity to methylmercury. Yakugaku Zasshi 127, 463-468.

Hwang, G.W., Hayashi, T., Kita, K., Takahashi, T., Kuge, S. and Naganuma, A. (2007): siRNA-mediated inhibition of phosphatidylinositol glycan Class B (PIGB) confers resistance to methylmercury in HEK293 cells. J. Toxicol. Sci., 32, 581-583.
Hwang, G.W., Wada, N., Kuge, S. and Naganuma, A. (2009): Overexpression of the novel F-box protein Ymr258c confers resistance to methylmercury in Saccharomyces cerevisiae. J. Toxicol. Sci., 34, 413-416.

Kahn, B.B., Alquier, T., Carling, D. and Hardie, D.G. (2005): AMPactivated protein kinase: ancient energy gauge provides clues to modern understanding of metabolism. Cell Metab., 1, 15-25.

Lee, J.Y., Hwang, G.W. and Naganuma, A. (2009): Rip1 enhances methylmercury toxicity through production of reactive oxygen species (ROS) in budding yeast. J. Toxicol. Sci., 34, 715-717.

Marsin, A.S., Bouzin, C., Bertrand, L. and Hue, L. (2002): The stimulation of glycolysis by hypoxia in activated monocytes is mediated by AMP-activated protein kinase and inducible 6-phosphofructo-2-kinase. J. Biol. Chem., 277, 30778-30783.

Naganuma, A., Miura, K., Tanaka-Kagawa, T., Kitahara, J., Seko, Y., Toyoda, H. and Imura, N. (1998): Overexpression of manganese-superoxide dismutase prevents methylmercury toxicity in HeLa cells. Life Sci., 62, PL157-161.

Sanders, M.J., Grondin, P.O., Hegarty, B.D., Snowden, M.A. and Carling, D. (2007): Investigating the mechanism for AMP activation of the AMP-activated protein kinase cascade. Biochem. J., 403, 139-148.

Usuki, F., Yasutake, A., Umehara, F., Tokunaga, H., Matsumoto, M., Eto, K., Ishiura, S. and Higuchi, I. (2001): In vivo protection of a water-soluble derivative of vitamin E, Trolox, against methylmercury-intoxication in the rat. Neurosci. Lett., 304, 199-203.

Watanabe, J., Nakamachi, T., Ogawa, T., Naganuma, A., Nakamura, M., Shioda, S. and Nakajo, S. (2009): Characterization of antioxidant protection of cultured neural progenitor cells (NPC) against methylmercury (MeHg) toxicity. J. Toxicol. Sci., 34, 315-325. 